\title{
Nonlinear response in polymer optical fibre Bragg grating based sensors
}

\author{
W. Zhang*a , D. J. Webb ${ }^{\mathrm{a}}$, and G. - D. Peng ${ }^{\mathrm{b}}$ \\ ${ }^{a}$ Aston Institute of Photonic Technologies, Aston University, Birmingham, B4 7ET, UK \\ ${ }^{\mathrm{b}}$ School of Electrical Engineering and Telecommunications, University of New South Wales, Sydney, \\ NSW 2052, Australia
}

\begin{abstract}
PMMA based polymer optical fibre Bragg gratings have been used for humidity, temperature and concentration sensing. Due to the water affinity of PMMA, the characteristic wavelength of the grating is largely modulated by the water content in the fibre. The rate of water transportation between fibre and surrounding depends on the permeability coefficient for PMMA, which is a function of surrounding temperature and humidity. This leads to increased water content with increasing humidity and temperature. Consequently the wavelength of the grating shows a nonlinear change over varying humidity and temperature. This nonlinearity needs to be calibrated prior to sensor application.
\end{abstract}

Key words: Polymers optical fibre, PMMA, fibre Bragg grating, humidity, temperature, concentration, Osmosis effect.

\section{INTRODUCTION}

Optical fibre has become an important part of sensor technology. Low loss light delivery, long interaction length, and compatibility with time/wavelength multiplexing are advantages which can build on technology developed for optical communications. Fibre Bragg grating (FBG) sensors inscribed in silica optical fibre have become an increasingly mature sensing technology. More recently, FBG sensors have been inscribed into poly(methyl methacrylate) (PMMA) based plastic optical fibre in both step-index and microstructured geometries. The physical and chemical properties of polymeric materials are rather different to silica and may offer advantages in certain situations. Polymer optical fibre Bragg gratings (POFBGs) are particularly appealing in medical devices as polymers are biologically compatible with human tissues and already routinely put in the human body in many applications. They have been shown to be more sensitive to both strain and temperature than is the case for silica FBGs [1]. An interesting feature of PMMA is the affinity for water which leads to a swelling of the fibre and an increase of index, both of which contribute to an increase in the Bragg wavelength of a FBG written in the fibre [2]. This is a potentially very useful property, which has possible applications in chemical processing, agriculture, food storage, paper manufacturing, semiconductor and pharmaceutical industries, where humidity is monitored and controlled to ensure product quality.

\section{CHARACTERISTIC WAVELENGTH OF POFBG}

The Bragg wavelength of a fibre grating depends on the effective core index, $n_{\text {eff }}$, and the grating pitch, $\Lambda$, both of which depend on the temperature $T$ and the water content $w$ in the case of a POFBG. For constant temperature the Bragg wavelength change is directly related to the water content inside the fibre. One can assume that the Bragg wavelength shifts linearly with water content in the fibre. Therefore the Bragg wavelength change can then be expressed in terms of

$$
\Delta \lambda=c w
$$

where $c$ is a coefficient associated with PMMA swelling, water dependence of the refractive index, and swelling induced stress.

For constant relative humidity $(\mathrm{RH})$ the Bragg wavelength change as a result of varying temperature, can be expressed as

$$
\Delta \lambda=\lambda_{B 0}(\alpha+\beta) \Delta T
$$

*w.zhang@aston.ac.uk 
where $\lambda_{B 0}$ is initial Bragg wavelength, $\alpha$, the thermal expansion coefficient, $\beta$, the thermo-optic coefficient. From equations (1) and (2) one can conclude that the wavelength change in POFBG sensors varies linearly with either humidity or temperature. Many reported results have shown such a tendency.

\section{EXPERIMENTAL RESULTS}

Experiments were carried out to inspect the response of a POFBG for humidity, temperature, and concentration sensing. POFBGs were fabricated by attaching a several centimetre length of POF to a single-mode silica fibre down-lead using UV curable glue. The PMMA based POF contained a $5 \mathrm{~mm}$ long FBG, fabricated by illuminating from above a phase mask placed on top of the POF using $325 \mathrm{~nm}$ UV light from a HeCd laser. A broadband light source with a total output optical power of $10 \mathrm{~mW}$ was used, launching light via a fibre circulator, into the POFBG under investigation. The reflection of the POF grating was monitored by using an IBSEN I-MON 400 wavelength interrogation system. In the experiments of temperature and humidity sensing the POFBGs were placed inside an environmental chamber (Sanyo Gallenkamp) so as to operate at the desired temperature and humidity.

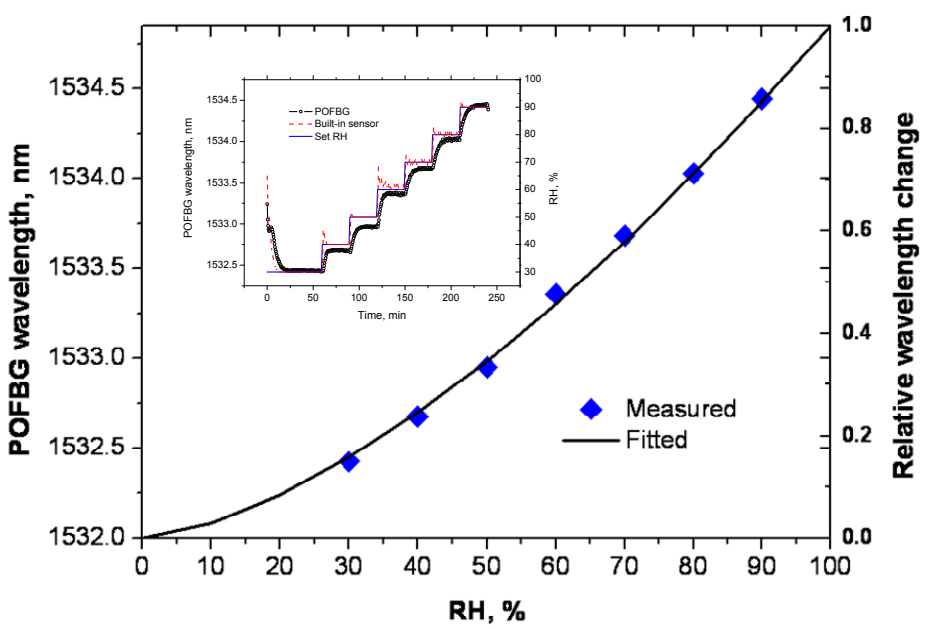

Fig. 1 POFBG wavelength vs. RH, and the fitted relative wavelength change vs. RH. Inset: Measured responses by the POFBG, the sensor built-in to the environmental chamber, and the set humidity value.

The environmental chamber was first programmed to change the relative humidity from $30 \%$ to $90 \%$ with an increment of $10 \%$ every $30 \mathrm{~min}$ while the temperature was fixed at $25^{\circ} \mathrm{C}$. A typical response of a POFBG is shown in Fig. 1 . The values of the set humidity and that measured by the chamber's built-in RH sensor are depicted in the figure as a comparison. The stabilized POFBG wavelength at each RH is depicted in Fig. 1. From it one can notice that the sensor shows a slightly nonlinear response against RH. Within a small RH range the wavelength of POFBG can be considered varying linearly with RH. Regression analysis on the whole range of RH data generated a relation between the wavelength change of the POFBG and the relative humidity, as illustrated in Fig. 1. It indicates a non-linearity, $m$, of 1.52. Therefore in this work the wavelength change against relative humidity, $H$, has a form of $\Delta \lambda \propto H^{m}$ [2].

A POFBG was also used to measure the concentration of saline solution [3]. In the experiment the POFBG was inserted into test tubes of saline solution with different concentrations. The reflection of the POF grating was monitored. Four samples of saline solution $(7.5 \%, 13 \%, 17.5 \%$ and $22 \%)$ were tested in order of ascending concentrations, then in order of descending concentration. The POFBG device was left for 60 minutes in each sample to allow equilibrium to be reached. The POFBG wavelength was monitored with an interval of 60 seconds. The wavelength change against concentration is plotted in Fig. 2. A typical captured response is shown in the inset of Fig. 2. From the captured response one can see that the POFBG wavelength varies significantly as a function of saline concentration. The POFBG wavelength decreases with increased saline concentration, and the process is reversible. From Fig. 2 one can see that the POFBG wavelength varies in a non-linear fashion with the concentration. It can be expressed by data regression as $\Delta \lambda \propto$ $-C^{m}$ with $m=1.25$. 


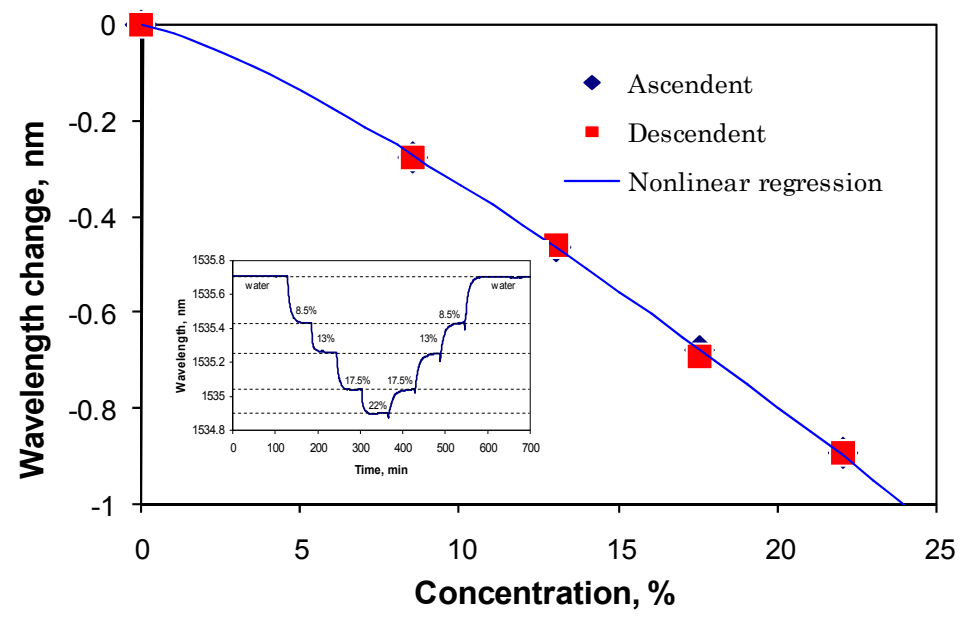

Fig. 2 Wavelength change of POFBG vs. concentration. Inset: measured POFBG response over time.

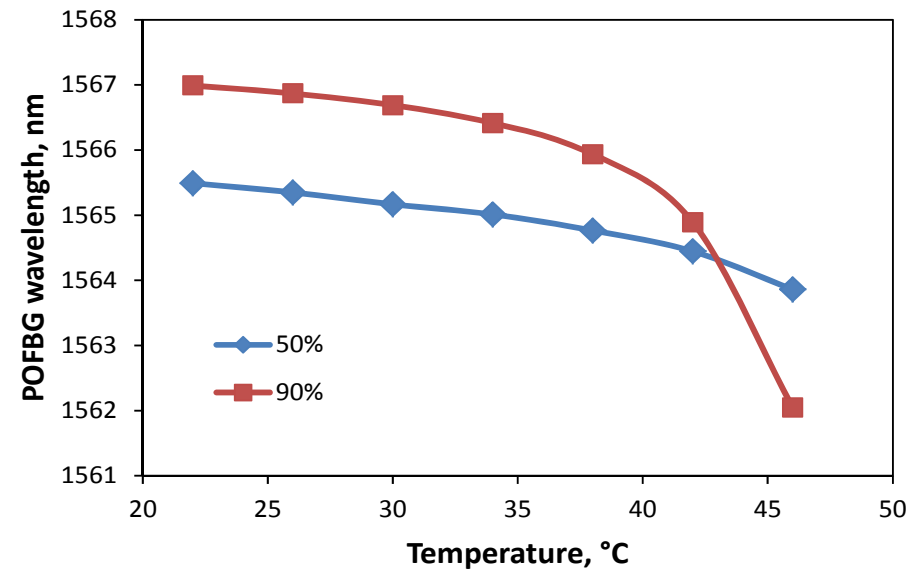

Fig. 3 Wavelength change of POFBG vs. temperature

Finally a POFBG was placed in the chamber under different temperature while relative humidity was fixed to investigate the temperature response of the POFBG sensors. The temperature responses of a POFBG sensor at different humidities are illustrated in Fig. 3. Two sets of data are presented for the relative humidity of $50 \%$ and $90 \%$, while the environmental chamber was programmed to change the temperature from $22{ }^{\circ} \mathrm{C}$ to $46{ }^{\circ} \mathrm{C}$ with an increment of $4{ }^{\circ} \mathrm{C}$ every $30 \mathrm{~min}$. From the results one can see that at a relative humidity of $50 \%$, the sensor response shows a slight nonlinearity. In the range of $22{ }^{\circ} \mathrm{C}$ to $38{ }^{\circ} \mathrm{C}$ the response exhibits a good linearity showing a sensitivity of $45 \mathrm{pm} /{ }^{\circ} \mathrm{C}$. At the relative humidity of $90 \%$, the sensor response exhibits a clear nonlinearity. Although at this relative humidity the response below $34{ }^{\circ} \mathrm{C}$ shows similar linearity and sensitivity to that at $50 \%$ relative humidity, the sensor exhibits a much higher sensitivity after $42{ }^{\circ} \mathrm{C}$. The temperature in the experiment was always kept below $50{ }^{\circ} \mathrm{C}$ as an irreversible response (annealing) may take place above $50^{\circ} \mathrm{C}$ in this POFBG.

\section{DISCUSSION AND CONCLUSION}

The responses of POFBGs for humidity, temperature and concentration have been investigated in this work. Unlike predicted by (1) and (2), the responses of POFBGs for these 3 parameters show nonlinearity. This is largely attributed to the water affinity of PMMA. As defined in equation (1) the Bragg wavelength of POFBG can be related to the absorbed water content in the fibre. For humidity sensing, when environmental humidity varies, the water diffuses into or out of the fibre, depending on the differential relative humidity between the fibre and its surrounding. This water diffusion can be defined by the diffusion theory of mass transfer [4] and the rate of water transportation between fibre and surrounding can be expressed as, 


$$
Q=\Delta P K_{w} S / d
$$

where $\Delta P$ is a differential hydraulic pressure, generated by the differential relative humidity inside and outside the fibre, $K_{w}$, the membrane permeability coefficient for water, $S$, the membrane area, and $d$, the membrane thickness. The larger rate of water transportation means more water content being diffused in or out of the fibre, thus more increase or decrease in wavelength change of the POFBG. The change in Bragg wavelength will stop once the differential humidity reaches a point of balance. Here permeability coefficient plays an important role and it is not constant at different humidities and temperatures [5]. Absorbed water has a plasticizing effect on some polymer materials and can lead to increased permeability. This leads to more water absorbed at higher relative humidity. Therefore the POFBG sensor shows a larger wavelength change in the higher relative humidity environment. Since the wavelength change is proportional to the water content in the fibre (2) can be re-written as $\Delta \lambda \propto H^{m}$ where $\mathrm{m}$ indicates the nonlinearity ( $m=1.52$ in this work).

In concentration sensing the sensor actually responds to the water concentration in the solution. Once solute exists in water the effect of osmosis takes place [6], generating an osmotic pressure inside the fibre. When the surrounding solution concentration increases, the osmotic pressure increases correspondingly to drive the water content in the fibre into the surrounding solution, consequently increasing the differential hydraulic pressure. This process will stop once there is a balance between the osmotic pressure and the differential hydraulic pressure. Therefore the water content in the polymer fibre depends directly on the solution concentration. Again equation (4) can be used to describe the rate of water transportation between fibre and surrounding, by replacing $\Delta P$ with a difference of $\Delta P$ and osmotic pressure. Since the osmotic pressure is proportional to the solution concentration, higher concentration generates a larger osmotic pressure thus a larger $\Delta P$, indicating a larger permeability coefficient. Therefore POFBGs shows a larger wavelength change at higher concentration.

In POFBG temperature sensing the wavelength change can be expressed by (3). A linear response can be expected if the thermo-optic coefficient and thermal expansion coefficient are constant. However, both coefficients are affected by water content $[2,7]$. In fact the temperature response of the POF can be explained by the direct thermal effects and the change in relative humidity due to the temperature change. With increasing temperature the water saturation pressure increases in air, thus the relative humidity is decreasing. This leads to a decrease of the water content in the POF and thus a decrease in POFBG wavelength. The variation of diffusion with temperature is an activated process [5]. Increases in temperature activate the polymer molecules creating voids by the movement of polymer segments which can be occupied by water molecules and thus the permeability coefficient increases with increasing temperature. This leads to larger wavelength change in POFBG sensors at higher temperature.

In conclusion the experimental results show that PMMA based POFBG sensors for humidity, concentration and temperature have nonlinear responses. This is mainly to do with the water transportation between fibre and surroundings in which the permeability coefficient increases with increasing humidity and temperature. To achieve a reliable operation POFBG sensor need to be calibrated prior to the application. Alternatively, for temperature and strain sensing, other polymers are available which do not exhibit the same affinity for water [8].

\section{REFERENCES}

[1] Liu, H. B., Liu, H. Y., Peng, G. D., and Chu, P. L.,, "Strain and temperature sensor using a combination of polymer and silica fibre bragg gratings, " Opt. Commun. 219, 139-142 (2003)

[2] Harbach, N. G., "Fibre bragg gratings in polymer optical fibres." PhD thesis Lausanne: EPFL (2008).

[3] Zhang, W., Webb, D. J., Peng, G.-D., "Polymer optical fibre Bragg grating acting as an intrinsic biochemical concentration sensor", Opt. Lett. 37(8), (2012)

[4] Crank, J., [The Mathematics of Diffusion]. 2nd ed., Clarendon Press - Oxford, 1975.

[5] Comyn, J., [Polymer Permeability], Elsevier Applied Science Publishers, London, (1985).

[6] van 't Hoff, J. H., "Osmotic pressure and chemical equilibrium," Nobel Prize lecture (1901)

[7] Drotning, W. D., and Roth, E. P., "Effects of Moisture on the Thermal-Expansion of Poly(Methylmethacrylate)," Journal of Materials Science 24(9): 3137-3140 (1989).

[8] Johnson, I. P., Yuan, W., Stefani, A., Nielsen, K., Rasmussen, H. K., Khan, L., Webb, D. J., Kalli, K., Bang, O., "Optical fibre Bragg grating recorded in TOPAS cyclic olefin copolymer," Electron. Lett. 47(4), 271-272 (2011). 